\title{
Prevalencia de Depresión en pacientes mayores de 60 años hospitalizados en el Servicio de Medicina Interna del Hospital Militar de Santiago
}

\section{Late life depression prevalence in patients admitted to an Internal Medicine Service}

Friedrich von Mühlenbrock S. ${ }^{1}$, Rocío Gómez D. ${ }^{2,3}$, Matías González V. ${ }^{2,3}$, Alberto Rojas A. ${ }^{2,3}$, Lorena Vargas G. ${ }^{2,3}$ y Christian von Mühlenbrock P. ${ }^{2,3}$

\begin{abstract}
Background: Depression (ICD-10 F33) is highly prevailing in general population, especially among elderly patients. It is associated with an increase of morbidity and mortality. In 1996, an study of elderly depression in the Military Hospital of Santiago showed a prevalence of 28,4\% in admitted patients. Objectives: To describe the prevalence of late life depression in patients admitted to an Internal Medicine Service. Methods: Patients were interviewed between 06-022009 and 09-06-2009. Inclusion criteria: Elder than 60 years old and admitted in the last 24 hours. Exclusion criteria: Pfeiffer $\geq 3$, Glasgow $<15$, aphasias, VAS $>5$, mental and terminal illness. Results: 131 patients were interviewed, 64 women and 67 men, mean age of 74,35 (DE 8,23 ) years old. Depression Prevalence was 22,13\% (29), 28,12\% (18) in women and 16,41\% (11) in men. Discussions: Late life depression in patients admitted to an Internal Medicine Service is common and often undetected or undertreated.
\end{abstract}

Key words: Depression, Elderly, Prevalence, Hospitalized, Internal Medicine. Rev Chil Neuro-Psiquiat 2011; 49 (4): 331-337

\section{Introducción}

Centiende por depresión a aquel estado de ániOmo triste que persiste, pese a haberse disipado la causa externa, o una expresión desproporcionada de ésta. Incluso, casos severos de depresión, no tienen causa externa precipitante ${ }^{2}$. El paciente con depresión pierde el interés, incluso de vivir, sintiéndose incapaz de realizar sus actividades pre- vias. Junto a la tristeza aparecen una serie de otros síntomas, como alteraciones del sueño, del apetito, del contenido del pensamiento y síntomas corporales, que interfieren gravemente con la calidad de vida ${ }^{3}$. Es un trastorno heterogéneo, de curso y respuesta variable al tratamiento y de mecanismos fisiopatológicos no comprendidos a cabalidad ${ }^{4}$. Se describe una prevalencia de depresión mayor de $10 \%$ en 12 meses y de 17\% a lo largo de la vida. En

Recibido: 25/05/2011

Aprobado: 17/10/2011

Psiquiatra y Médico Internista, Hospital Militar de Santiago.

Alumnos sexto año de Medicina Universidad de Los Andes.

Academia Científica de Estudiantes de Medicina, Universidad de Los Andes. 
Chile la prevalencia de episodio depresivo va entre $7,6 \%$ y $16,3 \%{ }^{5}$.

La depresión en el adulto mayor es más frecuente en mujeres, viudas, pacientes con enfermedades crónicas, en portadores de algún tipo de trastorno del sueño como insomnio, y en aquellos que han experimentado un evento estresante en sus vidas ${ }^{6}$, dismovilidad $^{7}$ y aislamiento social. Usualmente es subdiagnosticada ${ }^{8}$ y por ende sub tratada en los Servicios de Medicina, especialmente en hombres". La literatura indica que la depresión enmascarada, la depresión menor y la depresión secundaria a enfermedad somática tienen una mayor prevalencia en los ancianos que en gente joven ${ }^{10}$. Esto se debería según Sarkisian y colaboradores, a que la depresión es vista por la mayoría de los profesionales de la salud y por los propios pacientes, como parte normal del envejecimiento ${ }^{11}$. La clínica entre el anciano y el adulto no presenta mayores diferencias. Sin embargo, el anciano suele presentar más agitación psicomotora, compromiso cognitivo, delirios depresivos, ideas de pobreza y enfermedad física en relación a los menores de 60 años ${ }^{10}$. La pérdida de un ser querido, la coexistencia de varias enfermedades, el dolor de tipo crónico, alteraciones cognitivas y abuso de psicofármacos o alcohol serían los factores que con mayor frecuencia confunden para poder realizar un correcto diagnóstico ${ }^{12}$. La persistencia de este trastorno se asocia a una pobre calidad de vida, dificultad para comportarse adecuadamente del punto de vista social, mala adherencia al tratamiento, mala evolución de las enfermedades crónicas, aumento de morbimortalidad y de la tasa de suicidio ${ }^{13}$.

Un estudio (1996) realizado en el Hospital Militar de Santiago (HMS), por García y colaboradores, sobre la prevalencia de depresión en adultos mayores hospitalizados en el Servicio de Medicina Interna, reveló que el 28,4\% de éstos tenían depresión ${ }^{1}$.

El objetivo de este estudio es determinar la prevalencia de depresión en las personas mayores de 60 años que ingresan al servicio de Medicina Interna del HMS, utilizando la escala de depresión geriátrica de Yesavage-15 (versión abreviada de la de Yesavage-30 $)^{14}$. Es preciso aclarar que la OMS, determinó que el paciente geriátrico comienza desde los 60 años.

\section{Pacientes y Método}

\section{Pacientes}

Se realizó un estudio descriptivo en pacientes mayores de 60 años hospitalizados antes de cumplir 24 horas desde el ingreso al servicio de Medicina Interna del HMS. Los usuarios de este hospital pertenecen en su mayoría a pensionados de las fuerzas armadas y sus familiares (beneficiarios de la Caja de Previsión de la Defensa Nacional, CAPREDENA) así como público en general perteneciente a alguna Institución de Salud previsional (ISAPRE) o beneficiarios del Fondo Nacional de Salud (FONASA) en modalidad libre elección. Fueron excluidos del estudio los pacientes cuyo resultado en el test de Pfeiffer fue mayor o igual a 3 respuestas incorrectas (escala abreviada para diagnóstico de compromiso cognitivo), pacientes comprometidos de conciencia (Escala Glasgow menor a 15), pacientes con afasia, incapacidad de comprender instrucciones, pacientes con dolor crónico con Escala Visual Análoga (EVA) mayor a 5 y pacientes portadores de otros trastornos mentales (demencia y delirium mediante clínica y revisión ficha clínica) que impidiesen una correcta entrevista.

\section{Método}

Cinco estudiantes de pregrado de medicina de la Universidad de Los Andes, entrevistaron a pacientes mayores de 60 años que ingresaron al Servicio de Medicina Interna desde el 2 de junio hasta el 6 de septiembre de 2009. Las entrevistas fueron de lunes a sábado y se realizaron en todos los hospitalizados del servicio el mismo día de su ingreso o un día posterior a éste (en caso de haber ingresado un domingo).

Los alumnos fueron capacitados previamente por el psiquiatra del equipo para aplicar las escalas Pfeiffer y Yesavage-15, realizar una entrevista semiestructurada y detectar clínicamente demencia y delirium. A cada paciente que cumplió con los criterios de inclusión descritos, se le aplicó la escala de depresión geriátrica abreviada Yesavage- 15 además de una entrevista semiestructurada que incluyó datos personales previa firma de un consentimiento informado aprobado por el comité de ética del hospital. 
Aquellos pacientes con resultado positivo en el test de Yesavage-15, fueron evaluados a la mañana siguiente por el psiquiatra del equipo de investigación (entrevista psiquiátrica) para ratificar que no se tratara de un falso positivo y evaluar la necesidad de tratamiento. De los que obtuvieron resultado negativo se escogió uno de cada 5 pacientes aleatoriamente para ser evaluado clínicamente por el médico con el fin de descartar falsos negativos.

Se aplicó también la escala de Dismovilidad del Dr. Dinamarca ${ }^{7}$, para evaluar el grado de trastorno de la movilidad que presentaban los pacientes.

\section{Análisis estadísticos}

Se describen los datos obtenidos de las variables estudiadas. La prevalencia se obtuvo considerando como población total quienes cumpliesen con los criterios de inclusión y por depresión aquellos cuyo test Yesavage fuese mayor o igual a 6 puntos y estuviesen confirmados por el psiquiatra del equipo. Se calculó chi cuadrado para las variables estudiadas.

Los datos fueron analizados estadísticamente con el programa StatView ${ }^{\circledR}$ versión 5.0.1 (SAS Institute Inc.) y SPSS ${ }^{\circledR}$ versión 15.0.

\section{Resultados}

Durante el período de investigación ingresaron al Servicio de Medicina 585 pacientes. De éstos, 454 fueron excluídos (298 por ser menores de 60 años, 147 por patología médica o por cumplir con otros criterios de exclusión y 9 por rechazo explícito a la entrevista). Se entrevistaron 131 pacientes, de los cuales 64 eran mujeres $(48,85 \%)$ y 67 hombres $(51,15 \%)$. El rango de edad fue de 60 a 96 años de edad cumplidos, con un promedio de 74,35 años (Desviación Estándar (DE) $\pm 8,22$ ). De los evaluados, un 22,13\% (29) obtuvo un test de Yesavage positivo (diagnóstico de depresión), siendo un $28,12 \%$ (18) en mujeres y $16,41 \%$ (11) en hombres (Tabla 1); $\mathrm{p}=0,129$. Del total de positivos, el 89,6\% (26) se identificó en la categoría de depresión leve a moderada (puntaje mayor o igual a 6) y un 10,3\% (3) en la de depresión severa (puntaje mayor a 10). Tanto el puntaje promedio $3,63(\mathrm{DE} \pm 2,99)$ como la moda fue de 3 puntos. La totalidad de los resultados positivos fueron corroborados por el psiquiatra y ninguno de los negativos fue diagnosticado como depresión.

Según el nivel educacional, no se encontraron resultados positivos en los analfabetos, en los pacientes con nivel educacional básico incompleto fue de $31,58 \%$ (6), en los que cursaron toda la enseñanza básica 37,5\% (6), aquellos con educación media incompleta $30,43 \%$ (7), en educación media completa $14,29 \%$ (5), y en pacientes con estudios técnicos completos 17,39\% (4); p =0,368. No se evidenció depresión en los pacientes con estudios universitarios (tanto completos como incompletos) ni en aquellos con postgrado.

De acuerdo al estado civil, el grupo en que se encontró mayor prevalencia de depresión fue en los viudos, 38,46\% (15); el porcentaje de depresión tanto en solteros como en separados de hecho fue de $33,3 \%$ (1), mientras que en casados fue de $13,75 \%$ (11); $\mathrm{p}=0,035$.

Con respecto a la situación familiar, los paciente que vivían solos mostraron una prevalencia de $33,3 \%$ (6), aquellos que vivían con su familia 26,7\% (16), y los que vivían solamente con su cónyuge 10,2\% (5); $\mathrm{p}=0,045$.

$\mathrm{Al}$ interrogar sobre otros antecedentes personales, el porcentaje que relató consumir alcohol fue $42,98 \%$ (52). Dentro de este grupo la prevalencia de depresión fue de 17,3\% (9), mientras que en los que no ingerían alcohol, la prevalencia fue de $14,5 \%$ (10); $\mathrm{p}=0,135$. El porcentaje de pacientes que se declararon fumadores activos fue de 23,02\% (29), en este grupo la prevalencia de depresión fue de $27,59 \%$ (8); en cambio la prevalencia de depre-

Tabla 1. Interpretación Yesavage versus sexo

\begin{tabular}{|lrcc|}
\hline & $\begin{array}{c}\text { Yesavage } \\
\text { negativo } \\
\text { (recuento y } \\
\text { porcentaje) }\end{array}$ & $\begin{array}{c}\text { Yesavage } \\
\text { positivo } \\
\text { (recuento y } \\
\text { porcentaje) }\end{array}$ & Total \\
\hline Masculino & $55(83,3 \%)$ & $11(16,7 \%)$ & 66 \\
Femenino & $47(72,3 \%)$ & $18(27,7 \%)$ & 65 \\
Total & $102(77,9 \%)$ & $29(22,1 \%)$ & 131 \\
p & & & 0,129 \\
\hline
\end{tabular}


sión en los no fumadores fue de 20,62\% (20); p = 0,29 . El porcentaje de pacientes que no consumía ningún fármaco de manera habitual fue de 2,5\% (3), los pacientes con monofarmacia 19,2\% (23), polifarmacia menor (2-4 fármacos) $54,2 \%(65)$, polifarmacia mayor ( 5 o más fármacos) $24,17 \%$ (29). La prevalencia de depresión observada de acuerdo al consumo de fármacos fue de un $66,7 \%$ (2) en los pacientes que no consumían fármacos habitualmente, un $17,4 \%$ (4) en monofarmacia, $20 \%$ (13) en polifarmacia menor y de un $27,6 \%$ (8) en aquellos pacientes con polifarmacia mayor; $\mathrm{p}=0,85$.

Así mismo, se evaluó el grado de dismovilidad de los pacientes con la Escala de Dismovilidad del Dr. Dinamarca ${ }^{7}$, encontrándose un 73,02\% (92) en el grado $1 \mathrm{~A}$, un $12,7 \%$ (16) en $1 \mathrm{~B}, 6,35 \%$ (8) en $2 \mathrm{~A}$, $0,79 \%$ ( 1 ) en $2 \mathrm{~B}, 4,76 \%$ (6) en $3 \mathrm{~A}$ y $2,38 \%$ ( 3 ) en $4 \mathrm{~A} ; \mathrm{p}=0,078$. La prevalencia de depresión en los distintos grupos se muestra en la Tabla 2.

\section{Discusión}

Los trastornos del ánimo en los pacientes geriátricos hospitalizados tienen una importante prevalencia. García y cols, en $1996^{1}$ publicaron una prevalencia de $28,4 \%$ en pacientes mayores de 65 años hospitalizados en el HMS, cifra mayor a la obtenida en nuestro estudio. Esto puede explicarse en parte por la inclusión en este trabajo de pacientes entre los 60 y 65 años (acorde a la definición actual de paciente Geriátrico) y el uso de criterios de inclusión y exclusión más estrictos. A diferencia del estudio de 1996, en esta oportunidad se estudió solamente la prevalencia de depresión (CIE-10 F33) y se excluyeron a los pacientes con demencia (Pfeiffer $\geq 3$ ), aquellos con dolor EVA mayor a 5 y con enfermedad terminal para eliminar posibles factores de confusión.

Frecuentemente la depresión es subdiagnosticada ya que, en varias ocasiones, los síntomas depresivos se presentan de forma atípica o simplemente no son indagados dirigidamente por entenderse como un proceso natural del envejecimiento. Es por esto que consideramos relevante realizar el diagnóstico al ingreso para evidenciar el estado
Tabla 2. Interpretación Yesavage versus nivel de dismovilidad

\begin{tabular}{|lccc|}
\hline $\begin{array}{l}\text { Grado } \\
\text { dismovilidad }\end{array}$ & $\begin{array}{c}\text { Yesavage } \\
\text { Negativo }\end{array}$ & $\begin{array}{c}\text { Yesavage } \\
\text { Positivo }\end{array}$ & Total \\
1 A & 77 & 16 & 93 \\
1 B & 12 & 4 & 16 \\
2 A & 6 & 3 & 9 \\
3 A & 2 & 4 & 6 \\
4 A & 2 & 1 & 3 \\
Total & 99 & 28 & 127 \\
p & & & 0,057 \\
\hline
\end{tabular}

del paciente sin verse afectado por la estadía hospitalaria y procedimientos. Se vio que tan sólo 2 de los 131 pacientes tenían el diagnóstico actual de depresión y sólo 1 de ellos estaba en tratamiento. Esto repercute en gran medida en la calidad de vida y evolución de la enfermedad actual y basal, ya que una persona deprimida tiene mayores tasas de morbimortalidad y días de hospitalización. Esto se observó en un estudio de Almagro y colaboradores, donde se correlacionaba la mortalidad de los pacientes hospitalizados que padecen EPOC con el puntaje obtenido en el test de Yesavage- $15^{15}$.

Basados en las diferentes publicaciones, los pacientes hospitalizados poseen diversos factores de riesgo para presentar trastornos del ánimo ${ }^{16}$, entre ellos, la presencia de enfermedades crónicas como insuficiencia cardíaca o renal, enfermedades neoplásicas, accidente cerebrovascular, viudez, tabaquismo, escolaridad y el grado de dismovilidad. Es importante introducir este nuevo concepto en la práctica médica, ya que pacientes geriátricos presentan diversos niveles de limitación motora, desde dificultades en el traslado hasta estar encamado. En esta casuística hemos constatado que aquellos pacientes que permanecen la mayor parte del día sentados o encamados son los que presentan mayor tasa de depresión, sin embargo, no fue estadísticamente significativo, es decir, la muestra se distribuye homogéneamente para esta variable $(\mathrm{p}=0,057)$.

Al observar la proporción de pacientes con depresión según sexo, se evidencia una menor 
prevalencia de depresión en hombres, lo que no es estadísticamente significativo. Es importante señalar frente a este hecho, que los pacientes varones incluidos en el estudio, son en su mayoría miembros retirados de las Fuerzas Armadas, quienes se encuentran acostumbrados a los cambios de rutina diaria y ambiente, siendo un factor estresor de menor importancia que para la población civil el cambio desde el hogar al hospital.
Llama la atención que la prevalencia de depresión en este estudio, a diferencia de lo pensado, no aumenta conforme crece la edad, como se observa en la Figura 1. Por el contrario, con respecto a la escolaridad, observamos que a mayor nivel educacional, menor prevalencia, aunque no es estadísticamente significativo (Figura 2).

En relación al estado civil y depresión, ésta se manifestó con mayor prevalencia en pacientes
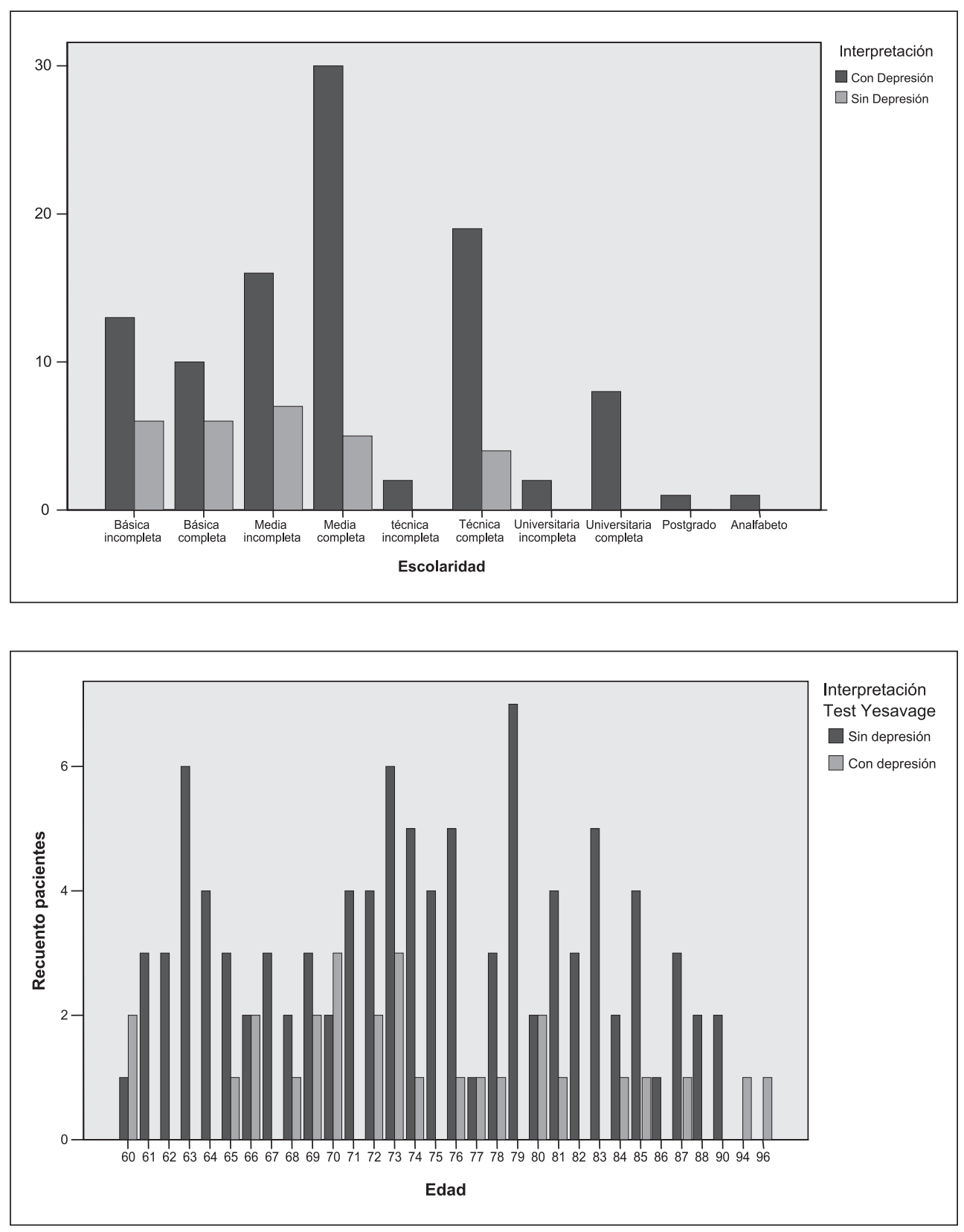

Figura 1. Interpretación test Yesavage versus nivel de escolaridad.
Figura 2. Interpretación test Yesavage versus edad. 
viudos, en concordancia con la literatura ${ }^{16}$, contrastando con los pacientes casados o convivientes, en quienes la depresión se manifiesta en menor proporción. Incluso, en relación a la compañía, los pacientes que relataron vivir solos presentaban mayor tasa de depresión que aquellos que viven en familia o con la pareja. Ambas variables son estadísticamente significativas, lo que puede hablar de la importancia de la condición de soledad en estos pacientes.

Con respecto al consumo de fármacos, se observó que el 78,37\% (94) de los pacientes tiene polifarmacia, lo que se coincide con otros estudios ${ }^{17}$. Aquellos con polifarmacia mayor tienen la más alta prevalencia de depresión en este estudio (no estadísticamente significativo, con una distribución homogénea de la depresión para esta variable). Esto se puede explicar por la relación existente entre polifarmacia y el número de patologías crónicas, siendo éste un factor predisponente para alteraciones del ánimo en el adulto mayor.

\section{Limitaciones del Estudio}

Es importante destacar que este estudio se realizó en el HMS y, por lo tanto, la población estudiada tiene características específicas, que pueden no reflejar completamente a la población adulta mayor en general. La mayoría son pacientes pertenecientes o relacionados con las Fuerzas Armadas, con una formación y previsión especial, entre otras cosas. Además, el tamaño de la muestra alcanzada no permite hacer un análisis confiable de todas las variables.

En suma, en este estudio, la depresión en los pacientes geriátricos hospitalizados, es frecuente y está subdiagnosticada. La importancia de ésta, según lo evidenciado en la literatura, radicaría en el tratamiento precoz que reducirá a mediano y largo plazo la morbimortalidad y los días de hospitalización de nuestros pacientes. Se requieren mayores estudios sobre la fisiopatología y tratamiento eficaz de esta enfermedad.

\section{Resumen}

Antecedentes: La depresión (ICD-10 F33) es de alta prevalencia en la población general, en especial entre los adultos mayores. Se asocia con un aumento de la morbilidad y la mortalidad. En 1996, un estudio de depresión en adultos mayores en el Hospital Militar de Santiago, mostró una prevalencia de 28,4\% en los pacientes admitidos. Objetivos: Describir la prevalencia de la depresión en pacientes adultos mayores admitidos en un Servicio de Medicina Interna. Métodos: Los pacientes fueron entrevistados entre el 2-06-2009 y el 6-09-2009. Criterios de inclusión: Mayores de 60 años y admitidos en las últimas 24 horas. Criterios de exclusión: Pfeiffer $\geq 3$, Glasgow < 15, afasias, VAS > 5, enfermedad mental y Terminal. Resultados: Se entrevistó 131 pacientes, 64 mujeres y 67 hombres, edad media de 74,35 (DE 8,23) años. La prevalencia de la depresión fue de 22,13\% (29), 28,12\% (18) en mujeres y 16,41\% (11) en hombres. Discusiones: La depresión en pacientes adultos mayores admitidos en un Servicio de Medicina Interna es común y a menudo no detectada o no tratada.

Palabras Claves: Depresión, adulto mayor, prevalencia, hospitalizado, medicina interna. 


\section{Referencias}

1. García C, Loyola JC, Armstrong T. Prevalencia de depresión en pacientes mayores de 65 años en pacientes hospitalizados en el Servicio de Medicina Interna del Hospital Militar de Santiago. Rev Med Chile 1996; 124: 431-6.

2. WHO Mental health: New understanding, new hope. The World Health Report 2001.

3. Katon WJ. Clinical and health services relationships between major depression, depressive symptoms, and general medical illness. Biol Psychiatry. 2003; 54: 216-26.

4. Belmaker RH, Agam G. Mechanisms of Disease: Major Depressive Disorder. N Engl J Med. 2008; 358: 55-68.

5. Retamal P. Trastornos Afectivos. En Florenzano R., Carvajal C., Weil K. Editores. Psiquiatría, Ed. Mediterráneo. 2008.

6. Bruce ML. Psychosocial risk factors for depressive disorders in late life.

7. Dinamarca JL. De la inmovilidad a la dismovilidad en geriatría. Ediciones de la Sociedad Chilena de Psicogeriatría. Tercera edición 2008.

8. Unützer J, Late-Life Depresion. N Engl J Med 2007; 357: 2269-76.

9. Klap R, Unroe KT, Unützer J. Caring for mental iones in the United Status: a focus on older adults. Am J Geriatr Psychiatry 2003; 11: 517-24.

10. Weil K. Psicogeriatría. En Florenzano R., Carvajal
C., Weil K. Editores. Psiquiatría, Ed. Mediterráneo. 2008.

11. Sarkisian Ca, Lee-Henderson MH, Mangione CM. Do depressed older adults who attribute depression to "old age" believe it is important to seek care? J Gen Intern Med 2003; 18: 1001-5.

12. Lebowitz BD, Pearson JL, Schneider LS, et al. Diagnosis and treatment of depression in late life: consensus statement update. JAMA 1997; 278: 1186-90.

13. National strategy for suicide prevention: goals and objetives for action. Rockville MD: Public Health Service, 2001.

14. Yesavage JA, Brink TL, Rose TL, et al. Development and validation of a geriatric depression screening scale: a preliminary report. J Psychiatr Res. 1982; 17 (1): 37-49.

15. Almagro P, Calbo E, Ochoa de Echagüen A, Barreiro B, Quintana S, Heredia JL. Mortality After Hospitalization for COPD. Chest 2002; 121: 14418 .

16. Bruce ML. Psychosocial risk factors for depressive disorders in late life. Biol Psychiatry 2002; 52: 175 84.

17. Jyrkkä J, Enlund H, Korhonen MJ, Sulkava R, Hartikainen S. Patterns of drug use and factors associated with polypharmacy and excessive polypharmacy in elderly persons: results of the Kuopio 75+ study: a cross-sectional analysis. Drugs Aging 2009; 26 (6): 493-503.

Correspondencia:

Dr. Freidrich von Mühlenbrock S.

Celular: 98766413

E-mail: sochisi@vtr.net 